\title{
In Situ Measurement of Thermal Diffusivity in Anisotropic Media
}

\author{
Tadeusz Kruczek • Wojciech P. Adamczyk • \\ Ryszard A. Bialecki
}

Received: 3 August 2012 / Accepted: 8 February 2013 / Published online: 12 April 2013

(C) The Author(s) 2013. This article is published with open access at Springerlink.com

\begin{abstract}
A knowledge of thermal conductivity/diffusivity is essential in several situations in engineering. This material property serves also as a measure of the quality of the manufactured materials. The thermal conductivity and diffusivity are measured by specialized labs using commercially available equipment. Even though both the number of such sites and the available measurement techniques are quite large, nondestructive, fast, and reliable techniques are still demanded. The developed technique, due to its rapidity and nondestructive character, can be embedded in a manufacturing process. As opposed to most methods, it does not require preparation of samples of a special shape (e.g., a small cylinder, a thin foil, cuboid). Moreover, one measurement cycle of the proposed technique yields two principal components of the diffusivities of orthotropic materials.
\end{abstract}

Keywords Anisotropic - Flash method - Thermal conductivity · Thermal diffusivity · Parker's method

\section{Abbreviations}

BVP Boundary value problem

FS Fundamental solution

T. Kruczek · W. P. Adamczyk $(\bowtie) \cdot$ R. A. Bialecki

Institute of Thermal Technology, Silesian University of Technology, 44-100 Gliwice,

Konarskiego 22, Poland

e-mail: wojciech.adamczyk@polsl.pl

T. Kruczek

e-mail: tadeusz.kruczek@polsl.pl

R. A. Bialecki

e-mail: ryszard.bialecki@polsl.pl 
GF

MS

TD
Green's function

Measurement surface

Thermal diffusivity

\section{Introduction}

A knowledge of thermal conductivity/diffusivity is essential in several situations in engineering. Simulation of heat conduction in solids, assessment of heat losses, checking the admissible temperature level, and calculation of thermal stresses are but a few examples of problems, where the value of the thermal conductivity is needed. This material property serves also often as a measure of the quality of the manufactured materials. Production of insulating or carbon materials are good examples of such a situation. Specialized labs offer the service of measurement of the thermal conductivity, using commercially available equipment. Even though both the number of such sites and the selection of measurement methods are quite large, non-destructive, fast, and reliable techniques are still under development. Moreover, no universal technique applicable to all materials, temperature ranges, and state of the matter of the material under investigation is known so far.

The current state of thermal diffusivity and conductivity measurements is described in [6] and [7] where advantages, disadvantages, and fields of application of the existing methods are described. This discussion will not be repeated here. Rather than that, a short overview of the available techniques which fall into three categories: steady-state, transient, and periodic are given.

Steady-state techniques require long time to produce the results. Maintaining the boundary external conditions (usually adiabatic) and the presence of contact resistance between the sample and the heater/cooler produce additional difficulties. Some techniques of this group additionally require a reference material. In oscillating fields techniques the probe is periodically heated either by a contact heater or by modulated heat radiation. The phase shift and amplitudes of the heater and temperature sensor are measured, from which the diffusivity can be evaluated. Some versions of this technique are patented [12,13]. The widely used Parker's flash method [16] is a transient-state technique whose great advantage is the short time of measurement. The shortcoming of the technique is that it is destructive and it is based on a 1D model. The treatment of anisotropic (orthotropic) materials require thus more separate probes and experiments. The technique has been extended to deal with anisotropic medium [9] but still the method cannot be applied in situ, as the measurements are carried out using a small cuboidal sample. Nonetheless, Parker's technique with its numerous extensions is widely recognized as a reliable measuring technique. Commercial measurement equipment where this technique is implemented is available. The technique is also a recommended standard for measuring thermal diffusivity [10]. The technique is still improved [3] by accounting for the heat losses, nonhomogeneous heat distribution of the laser beam, etc.

The majority of the known methods are destructive, i.e., they require preparation of a sample of a special shape (e.g., a small cylinder, a thin foil, cuboid). Moreover, 
except for the Parker methods, the measurement procedures are long and can be carried out only in a lab environment.

Another group of measurement techniques is the active thermography, also known as lock-in thermography. These groups of methods, originally developed as means of detecting damages/flaws in materials $[14,18]$ consists of analyzing the dynamic thermal response of a material subjected to a time varying heat source. Reference [5] describes a technique where the time-dependent temperature field is generated by periodic heat source located on the surface, while the temperature field is measured by an IR camera looking at the same surface. Reference [19] discusses the theory of another variant of the lock-in thermography; however, no experimental results of the technique are reported.

\section{Description of the Proposed Technique}

The paper presents a new method of determining the thermal diffusivity (TD) of anisotropic materials. The technique is non-destructive and allows for in situ measurements. The entire time of the measurement and evaluation of the results is of the order of a minute. Due to these features, the technique may be used for on-line property measurements that are embedded in the manufacturing processes. The TD is retrieved by processing of the temporal and spatial distribution of the temperature field recorded by a fast infrared (IR) camera. The perturbations of the temperature field are inducted by a short laser impulse absorbed within a small surface area of the sample. The energy of the pulse should be able to generate a temperature rise of the order of $10^{\circ} \mathrm{C}$ a few millimeters from the point where the laser ray impinges the material. A characteristic feature of the developed technique is that, as opposed to the standard Parker [16] method, the laser and the IR camera are located on the same side of the sample, see Fig. 1. Such a configuration is known in the literature as a front face technique.

The proposed technique can be applied to both orthotropic and isotropic materials of one plane surface. The laser impulse impinges that surface, further referred to as the measurement surface (MS). The temperature field on this surface is recorded by

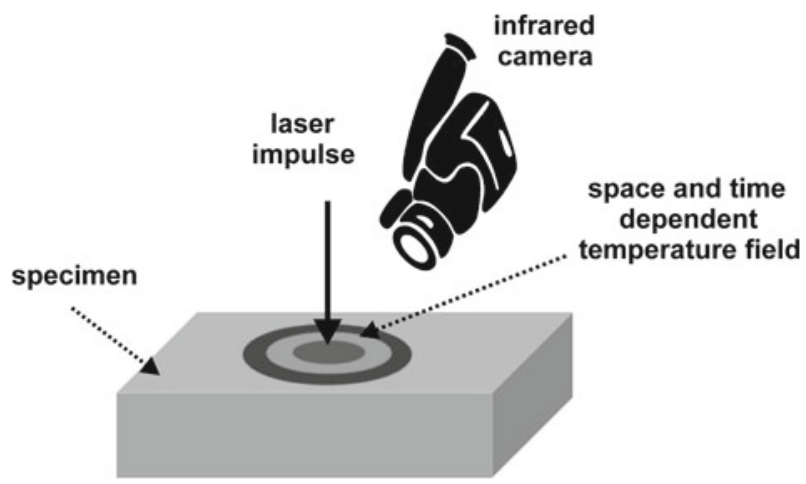

Fig. 1 Schematic of the method 
the camera. One laser shot produces data to retrieve two principal components of the TD tensor being parallel to the MS. The technique can be used to retrieve the TD or thermal conductivity. In the latter case, the density and the specific heat should be determined by separate measurement. The evaluation of the TD is carried out in two stages. In the first, the recorded temperature field is processed to find the shape of the isotherms. At this stage, the recorded isotherms are fitted to their theoretical, elliptic shape. The result of this process is the ratio of the principal components of the TD. In the second stage, the temporal variation of the ratio of temperatures at a collection of points is evaluated. Application of a simple inverse technique produces then one principal component of the TD tensor. The second component is obtained from the previously determined ratio of the two TD tensor components. So far the technique has been tested for media whose thermal conductivities were in the range between $5 \mathrm{~W} \cdot \mathrm{m}^{-1} \cdot \mathrm{K}^{-1}$ and $40 \mathrm{~W} \cdot \mathrm{m}^{-1} \cdot \mathrm{K}^{-1}$. Extension to lower conductivities presents no problems. Higher values would most probably require a modification of the equipment and/or the model.

\section{Mathematical Model}

When deriving the equation of transient heat conduction in the anisotropic body, the following assumptions have been made:

- The specimen is a semi-infinite, orthotropic body of constant material properties.

- The laser impulse is treated as a pointwise and instantaneous heat source located on the boundary (MS) of the semi-infinite body.

- With the exception of the point where the pointwise heat source is acting, the plane $z=0$ is adiabatic.

- Two principal components of the thermal conductivity (TD) tensor are in plane with the MS.

- The emissivity of the outer surface should be high enough to prevent significant laser beam reflection during measurements.

The solution of such a boundary value problem (BVP) is known as Green's function (GF) for a semi-infinite domain with a Neumann boundary condition and the source located at $z=0$. References [4,8] give a detailed discussion of the GF method employed to the thermal conduction equation.

The solution of the relevant BVP can be constructed starting from the differential equation of transient heat conduction in an infinite orthotropic solid with a unit, instantaneous, pointwise heat source located at $x=\xi, y=\eta, z=\zeta$, at time instant $t=\tau$,

$$
\lambda_{x} \frac{\partial^{2} T}{\partial x^{2}}+\lambda_{y} \frac{\partial^{2} T}{\partial y^{2}}+\lambda_{z} \frac{\partial^{2} T}{\partial z^{2}}+\delta(x-\xi, y-\eta, z-\zeta ; t-\tau)=c \rho \frac{\partial T}{\partial t}
$$

where $T$ stands for temperature, $x, y, z$ are the ortho Cartesian coordinates, $t$ denotes time, $\lambda_{x}, \lambda_{y}, \lambda_{z}$ are principal conductivities in $x, y, z$ directions, $c$ and $\rho$ stand for specific heat and density, and $\delta$ denotes Dirac's impulse function. Denoting 
$k_{x}=\lambda_{x} / \lambda_{x}, k_{y}=\lambda_{y} / \lambda_{x}, k_{z}=\lambda_{z} / \lambda_{x}$ and redefining the spatial variables $x=\bar{x} \sqrt{k_{x}}, y=\bar{y} \sqrt{k_{y}}$, and $z=\bar{z} \sqrt{k_{z}}$ brings Eq. 1 to

$$
\frac{\partial^{2} T}{\partial \bar{x}^{2}}+\frac{\partial^{2} T}{\partial \bar{y}^{2}}+\frac{\partial^{2} T}{\partial \bar{z}^{2}}+\bar{\delta}(\bar{x}-\bar{\xi}, \bar{y}-\bar{\eta}, \bar{z}-\bar{\zeta} ; t-\tau)=\frac{1}{D_{x}} \frac{\partial T}{\partial t}
$$

where $D_{x}=\lambda_{x} / c \rho$ is the TD component in $x$ direction and $\bar{\delta}$ is the Dirac delta in the new coordinates system defined as $\bar{\delta}=\delta /|J|$ where $|J|=\sqrt{k_{x} k_{y} k_{z}}$ is the Jacobian of the coordinate transformation. The presence of the Jacobian results from the requirement that the integral of the Dirac delta should be equal to one, both in the old and new coordinate systems [11].

$$
\begin{aligned}
& \int_{V}\left[\int_{0}^{\infty} \delta(x-\xi, y-\eta, z-\zeta, t-\tau) \mathrm{d} t\right] \mathrm{d} x \mathrm{~d} y \mathrm{~d} z \\
& =\int_{V}\left[\int_{0}^{\infty} \bar{\delta}(\bar{x}-\bar{\xi}, \bar{y}-\bar{\eta}, \bar{z}-\bar{\zeta}, t-\tau) d t\right]|J| \mathrm{d} \bar{x} \mathrm{~d} \bar{y} \mathrm{~d} \bar{z}=1
\end{aligned}
$$

where $V$ stands for the integration domain in the original and transformed coordinates frame. For a homogeneous initial condition and infinite domain solution of Eq. 2 bears the name of the fundamental solution (FS) of the heat conduction equation and takes the form,

$$
T(\bar{x}, \bar{y}, \bar{z}, t-\tau)=\frac{\exp \left[-\frac{(\bar{x}-\bar{\xi})^{2}+(\bar{y}-\bar{\eta})^{2}+(\bar{z}-\bar{\zeta})^{2}}{4 D_{x}(t-\tau)}\right]}{8 c \rho \pi^{3 / 2}\left[D_{x}(t-\tau)\right]^{3 / 2} \sqrt{k_{x} k_{y} k_{z}}}
$$

If the energy of the impulse is $q$ and the initial condition $T_{\text {init }}$, the solution in the original coordinate system turns to

$$
T(x, y, z, t-\tau)=\frac{q \exp \left[-\frac{(x-\xi)^{2} / k_{x}+(y-\eta)^{2} / k_{y}+(z-\zeta)^{2} / k_{z}}{4 D_{x}(t-\tau)}\right]}{8 c \rho \pi^{3 / 2}\left[D_{x}(t-\tau)\right]^{3 / 2} \sqrt{k_{x} k_{y} k_{z}}}+T_{\text {init }}
$$

The solution in the semi-infinite medium with insulation on $z=0$ can be obtained by a superposition of two pointwise instantaneous heat sources acting at $\zeta= \pm L$, i.e., located symmetrically with respect to the $z=0$ plane and acting at time $\tau=0$. The temperature resulting from the presence of such two sources in an infinite medium is a sum of two-shifted solutions of Eq. 5: 

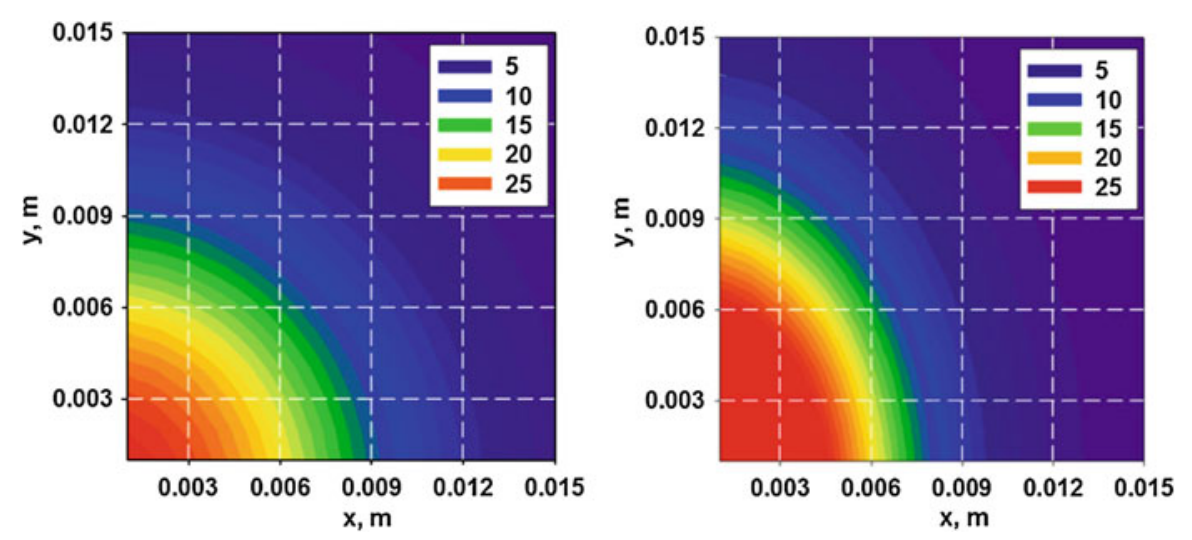

Fig. 2 Isotherms for $k_{y}=1$ (left) are circles, and (right) for $k_{y}=2.0\left(\rho=1600 \mathrm{~kg} \cdot \mathrm{m}^{-3}, c=\right.$ $\left.\left.900 \mathrm{~J} \cdot \mathrm{kg}^{-1} \cdot \mathrm{K}^{-1}\right), q=100 \mathrm{~J}, t=3 \mathrm{~s}\right)$

$$
\begin{aligned}
T(x, y, z, t)= & \frac{q \exp \left[-\frac{(x-\xi)^{2} / k_{x}+(y-\eta)^{2} / k_{y}+(z-L)^{2} / k_{z}}{4 D_{x} t}\right]}{8 c \rho \pi^{3 / 2}\left[D_{x} t\right]^{3 / 2} \sqrt{k_{x} k_{y} k_{z}}} \\
& +\frac{q \exp \left[-\frac{(x-\xi)^{2} / k_{x}+(y-\eta)^{2} / k_{y}+(z+L)^{2} / k_{z}}{4 D_{x} t}\right]}{8 c \rho \pi^{3 / 2}\left[D_{x} t\right]^{3 / 2} \sqrt{k_{x} k_{y} k_{z}}}+T_{\text {init }}
\end{aligned}
$$

Taking the limit $L \rightarrow 0$ brings the temperature in the plane $z=0$ to a form,

$$
T(x, y, z=0, t)-T_{\text {init }}=\frac{q \exp \left[-\frac{(x-\xi)^{2} / k_{x}+(y-\eta)^{2} / k_{y}}{4 D_{x} t}\right]}{4 c \rho \pi^{3 / 2}\left[D_{x} t\right]^{3 / 2} \sqrt{k_{x} k_{y} k_{z}}}
$$

Figure 2 illustrates the influence of the anisotropy on the shape of the isotherms. The temperature fields have been calculated from Eq. 7 taking the ratio of the principal thermal conductivities $k_{y}$ equal to 1 and 2.0, respectively.

It can readily be seen, that for any choice of temperature $T(x, y, z=0, t)=T_{\text {iso }}$ and time $t=t_{\mathrm{c}}$ the isotherms are ellipses whose equation is

$$
\frac{(x-\xi)^{2}}{C D_{x}}+\frac{(y-\eta)^{2}}{C D_{y}}=1
$$

where $C$ is a constant defined as

$$
C=-4 k_{x} t_{\mathrm{c}} \ln \left[\frac{4 c \rho \pi^{3 / 2}\left(D_{x} t_{\mathrm{c}}\right)^{3 / 2} \sqrt{k_{x} k_{y} k_{z}}\left(T_{\text {iso }}-T_{\text {init }}\right)}{q}\right]
$$



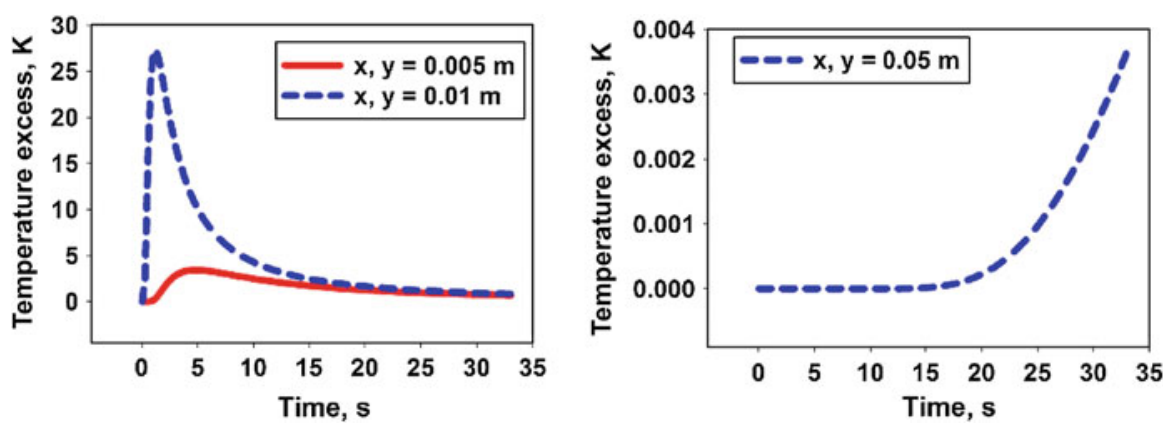

Fig. 3 Temporal variation of the temperature excess at selected points on the measurement surface $z=0$ for $\rho=1600 \mathrm{~kg} \cdot \mathrm{m}^{-3}, c=900 \mathrm{~J} \cdot \mathrm{kg}^{-1} \cdot \mathrm{K}^{-1}$, thermal conductivity $\lambda_{y}=11 \mathrm{~W} \cdot \mathrm{m}^{-1} \cdot \mathrm{K}^{-1}$, the ratio of thermal conductivities $k_{y}=1.2$, and the energy of the pulse $q=100 \mathrm{~W}$

Taking into account that $k_{x}=1$, the ratio of the semi-axes $a$ and $b$ of the ellipse is

$$
k_{y}=\left(\frac{b}{a}\right)^{2}=\frac{\lambda_{y}}{\lambda_{x}}=\frac{D_{y}}{D_{x}}
$$

where $a, b$ represent the $x$ and $y$ semi-axes, respectively. Equation 10 shows that the ratio of the principal components of the thermal conductivity/diffusivity tensor in $x$ and $y$ directions can be assessed by measuring the semi-axes of instantaneous isotherms. The procedure of evaluation of this quantity is given in the next section.

The propagation of the heat within the body results in a characteristic shape of the temporal variation of the temperature excess at each point on the MS. Figure 3 shows a plot of the temperature excess versus time for three points located on this surface. It can be seen that for locations close to the heat source, the temperature assumes a maximum within a few seconds. For points located further away, the temperature change is very small, which suggests that for the data used when plotting the temperature variation, even not very massive bodies can be treated as infinite media. A general rule [8] states, that a finite body of dimension $H$ can be treated as a semi-infinite one when the boundary conditions at $H$ do not influence the temperature near the point of observation located at $H=0$. This happens always when the time of observation is short enough, i.e., the Fourier number based on characteristic dimension $H$ is less than 0.05 [8].

The temperature field defined by Eq. 7 depends on $q$, a difficult to assess portion of the energy emitted by the laser that is absorbed by the body. This energy cannot be measured directly, because part of the energy is reflected from the measurement surface. Moreover, the temperature field depends also on the unknown thermal conductivity ratio $k_{z}$. To remove both $q$ and $k_{z}$ from the final relationship, an auxiliary variable $\Theta$ is introduced. This quantity is defined as a ratio of two temperature excesses over the initial temperature. Both excesses are evaluated at the same point $x_{i}, y_{i}$, but each correspond to different times $t_{1}, t_{2}$. An explicit relationship defining the ratio reads 


$$
\begin{gathered}
\Theta_{\text {model }}\left(x_{i}, y_{i}, \xi, \eta, t_{1}, t_{2}, k_{y}, D_{x}\right)=\frac{T\left(x_{i}, y_{i}, z=0, t_{1}\right)-T_{\text {init }}}{T\left(x_{i}, y_{i}, z=0, t_{2}\right)-T_{\text {init }}} \\
=\frac{\sqrt{t_{2}^{3}}}{\sqrt{t_{1}^{3}}} \exp \left[\frac{1}{4 D_{x} k_{y}}\left(k_{y}\left(x_{i}-\xi\right)^{2}+\left(y_{i}-\eta\right)^{2}\right)\left(\frac{1}{t_{2}}-\frac{1}{t_{1}}\right)\right]
\end{gathered}
$$

As already mentioned, the ratio of thermal conductivities $k_{y}$ can readily be evaluated by measuring the geometry of the isotherms. Once this quantity is determined, Eq. 11 can be used to calculate the thermal diffusivity $D_{x}=\lambda_{x} /(c \rho)$. The diffusivity along the $y$-axis is then simply $D_{y}=D_{x} k_{y}$. In practice, using Eq. 11 to determine the thermal diffusivity leads to poor accuracy, as the problem at hand is ill-conditioned. The two step process of retrieving the diffusivity from temperature measurements is addressed in the next section.

\section{Evaluation of the Diffusivity from Measurements}

The temperature is measured using an infrared camera that records both temporal and spatial variation of the temperature in the vicinity of the pulse. The details of the measurement equipment can be found in Appendix 1.

Intensities of radiation leaving the MS are recorded by pixels of the IR camera at certain time instants. Each pixel collects the intensity leaving a small square located on the MS. These intensities are transformed to temperatures whose values are assigned to the centers of the mentioned squares. In the first step of the calculations, the location of the isotherms is determined by bilinear interpolation between the four recorded pixels. The idea of this approach is shown in Fig. 4.

As a result of this procedure, a set of points corresponding to the selected value of the isotherm is obtained. Figure 5 shows a sample set of such points. The (blue) squares stand for pixels where the temperatures bracketing the isotherm are recorded. The (red) circles denote the interpolated locations of the isotherm.

Due to the measurement error and local fluctuations of material properties, the isotherms do not have an ideal elliptic shape. A least-squares fit of the measured ellipse to the theoretical one, produces not only the semi-axes of the ellipse $a, b$, but also the location of its center $\xi, \eta$. For $N$ isotherm points, this optimization problem is defined as

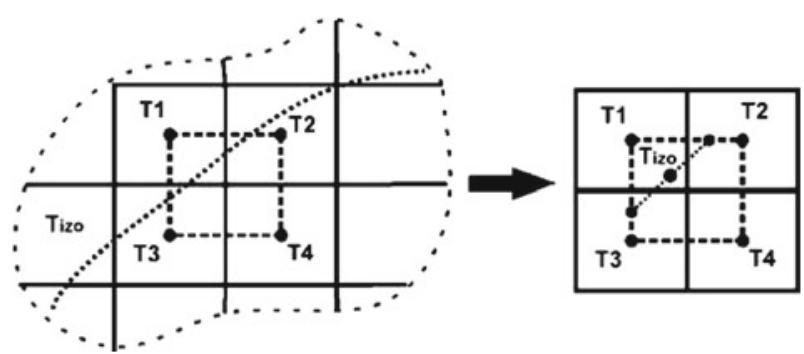

Fig. 4 Determining the coordinates of the isotherm by bilinear interpolation 
Fig. 5 Evaluated isotherm based on bilinear interpolation

Fig. 6 Fitting experimental and theoretical isotherms
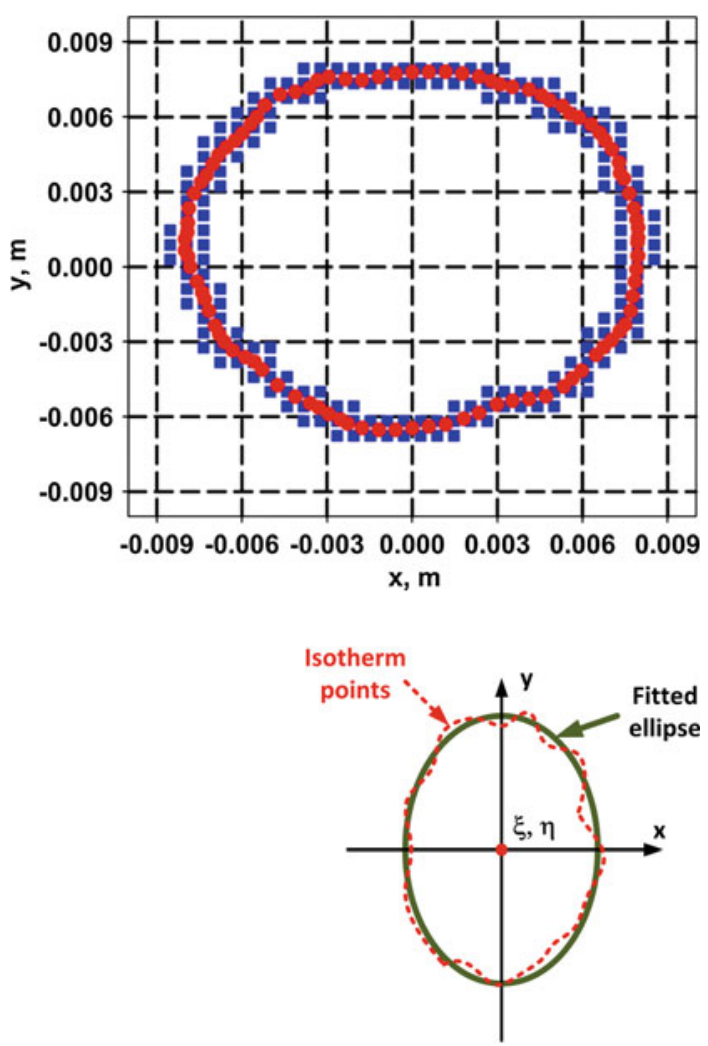

$$
\min _{a, b, \xi \eta} \sum_{i=1}^{N}\left[\frac{\left(x_{i}-\xi\right)^{2}}{a^{2}}+\frac{\left(y_{i}-\eta\right)^{2}}{b^{2}}-1\right]
$$

The above nonlinear programming problem is solved using the LevenbergMarquardt technique [17]. A sample result is shown in Fig. 6 where the dotted and solid lines represent the measured and theoretical isotherm shapes.

Once the semi-axes and the coordinates of the center of the ellipse are evaluated, the ratio of the thermal conductivities $k_{y}=\lambda_{y} / \lambda_{x}$ can readily be determined from Eq. 10.

In the next step, the thermal diffusivity $D_{x}$ is determined. This is accomplished by comparing two temperatures measured at two time instances with temperature ratios calculated from Eq. 11. The set of points used for this comparison is that, which was used to determine the elliptic shapes of the isotherms. The evaluation of the diffusivity is carried out minimizing the discrepancy of the measured and theoretical values of the ratio of temperature excesses,

$$
\min _{D_{x}} \sum_{i=1}^{N}\left[\Theta_{\operatorname{model}}\left(x_{i}, y_{i}, \xi, \eta, t_{1}, t_{2}, k_{y}, D_{x}\right)-\Theta_{\text {measured }}\left(x_{i}, y_{i}, t_{1}, t_{2}\right)\right]^{2}
$$


So defined least-squares problems require additional stabilization provided by the Levenberg-Marquardt technique [17]. It should be stressed that the center of the laser beam is not measured directly, but is obtained from isotherm fitting as defined in Eq. 12. Moreover, although the model temperature field is singular at $(x=\xi, y=\eta, z=0)$, this point does not enter the calculations, as the temperatures used in the inverse procedure are measured at a certain distance from the center of the laser beam.

Evaluation of material properties is an inverse problem where the measured temperatures (or less frequently, heat fluxes) are used to retrieve the thermal diffusivity, conductivity, or specific heat. Inverse problems are known from their ill-posedness [2], which means, that unless special measures are taken, small errors in the input data (e.g., measurement errors) are amplified in the course of calculations. To minimize this instability, several techniques can be used. In this study, the Levenberg-Marquardt technique provides a necessary regularization. An overview of other available approaches can be found in relevant literature on inverse problems, e.g., $[2,15]$ and works cited therein.

\section{Experiment}

The basic idea of the experiment involves recording the temperature field excited by the high power laser impulse impinging on the surface of the specimen. A schematic of the rig is shown in Fig. 7.

The laser optical head is connected with the laser by an optical fiber and the IR camera are mounted in a rectangular bin open from the bottom, further referred to as a container. The role of the container is to isolate the MS from external influence caused by radiation and air movement. As the IR camera measures the intensity of radiosity (sum of emitted and reflected radiation), each external source of radiation (e.g., reflected sun ray, hot surfaces in the vicinity of the rig) may introduce serious error in the measurement. Similarly, air movement in the large industrial shop floor where the measurement rig is installed is highly probable. Though air movement would not influence the temperature measurement, it would cause additional convective heat flux on the boundary. As the developed model assumes adiabatic conditions, the forced convection triggered by the air movement would also introduce additional error.

At the beginning of the experiment, the container is settled on the MS of the sample. The laser beam and the IR camera optical axis are coaxial and perpendicular to the MS. The reason for such an arrangement is obvious. In the mathematical model of the heat transfer, a pointwise heat source is assumed. A circular heat source of small diameter produced by a focused laser beam whose direction is normal to the measurement plane, is a good approximation of such a source. A tilted laser beam would produce an elliptic heat source on the measurement surface. As the ellipticity of the isotherms are important measured parameters, any deviation from the circular shape of the heat source would significantly deteriorate the accuracy of the measurement.

The IR camera records subsequent snapshots of the temperature field. If the optical axis of its lens is not perpendicular to the measurement surface, the recorded temperature field should be projected on the measurement surface. Such a transformation would produce additional error. The laser head and the IR camera share therefore 


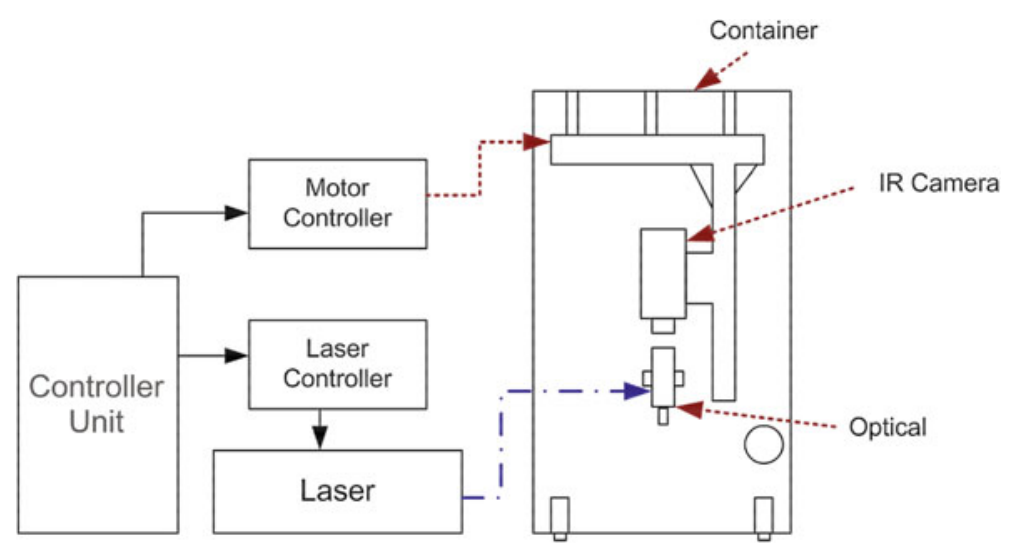

Fig. 7 Setup of the experimental rig

the same optical axis. In such a configuration the field of view of the IR camera is blocked by the laser head. To avoid this, a system of linear motors and guide bars is installed in the container. Their role is to remove, after the flash, the laser head from the field of view of the IR camera. The optical head is moved with a high speed of approximately $10 \mathrm{~m} \cdot \mathrm{s}^{-1}$ which ensures that the temperature can be recorded by the camera $0.3 \mathrm{~s}$ to $0.4 \mathrm{~s}$ after the flash.

To control the laser flash temperature recording movement of the laser head, an in-house PC application written in National Instrument LabVIEW 2011 has been developed. All devices are connected by a serial bus (laser, motors) and Ethernet link (IR camera). The serial bus is used by the laser and step motor controller, whereas the Ethernet connection is used by the IR camera.

\section{Numerical Calculations}

After the measurement procedure is finished, the calculation part of the application is activated. It starts with a transformation into temperatures of the intensities of radiation recorded by the pixels of the IR camera. This numerically intensive procedure is performed in parallel on a multicore processor. In the next step, the isotherms are selected, from the shape of which, the ratio of the thermal conductivities (diffusivities) is determined. Once this is done, from the ratio of the temperature excess at given points and two selected times, the diffusivity in the $x$ direction is evaluated.

The IR camera produces a huge amount of data, taking snapshots of the temperature field at high frequency. Only very few recorded data are needed to retrieve the required material data. To determine the ratio of the thermal conductivities from Eq. 12, the time at which the snapshot of temperature is taken, should be defined. Similarly, evaluation of the thermal diffusivity from Eq. 13 requires a definition of two time instants $t_{1}, t_{2}$ at which the excess temperatures are taken. To simplify the analysis, it has been assumed that the time at which the elliptic shapes of the isotherms are approximated using Eq. 12, is identical with the time $t_{1}$ used to evaluate the diffusivity. The locations $x_{i}, y_{i}$ used in Eq. 13 are the same as those used to determine the ellipticity of isotherms from Eq. 12. 
The selection of times and locations where the temperature field is sampled, is a quite involved problem. At the present stage of the development of the technique, this problem is treated to some extent heuristically using also some elements of Bayesian reasoning. A sound approach based on the comparison of the model and a CFD simulation encompassing the influence of convection and radiation is under development. The values given in this section depend on the spatial and temporal resolution of the IR camera used in the investigations. The parameters of the device are described in Appendix 1, whereas the uncertainty analysis for IR camera measurement is provided in Appendix 2.

The selection of the temperatures is subject to several constraints. The model assumes a pointwise, instantaneous heat source. In reality, the laser flash produces a heat source of finite dimensions generating heat within a finite time. Moreover, the distribution of heat within the laser spot is not uniform. Thus, both the time and location of the isotherms used to determine their ellipticity should be far enough in time and space from the laser spot. The time $t_{2}$ entering Eq. 13 should not be too far from the time of the pulse, as then the temperature rapidly decays in time. As a result, for long times, the error of the temperature measurement might be comparable with the actual temperature reading. Moreover, at later times, the influence of the convective and radiative heat exchange, neglected in the model, might be significant.

The first temperature field that can be recorded by the camera is that when the laser head is moved away from the field of view of the IR camera. Starting from this point, the temperature field is recorded at a frequency defined by that the camera used. Each of the recorded fields can be considered as the first time instant $t_{1}$ that is used to determine the ellipticity of the isotherms and simultaneously is used in Eq. 11 to evaluate the thermal diffusivity. For materials with a thermal conductivity between $5 \mathrm{~W} \cdot \mathrm{m}^{-1} \cdot \mathrm{K}^{-1}$ and $40 \mathrm{~W} \cdot \mathrm{m}^{-1} \cdot \mathrm{K}^{-1}$, the upper bound of time $t_{1}$ is heuristically taken at the level of $1.1 \mathrm{~s}$ after the first recorded temperature field. The interval between the upper and lower bound of $t_{1}$ is sampled every $0.1 \mathrm{~s}$. For each value of the time, random values of isotherms are generated in the interval between $3 \mathrm{~K}$ and $13 \mathrm{~K}$ above the initial temperature. The final set of isotherms should fulfill the following conditions (values depend on the spatial and temporal resolution of the IR camera). The minimum difference between the isotherms should be at least $0.3 \mathrm{~K}$. The number of pixels taken to interpolate the isotherm should be at least 60 . The reason for accounting of the first condition is obvious; close isotherms do not produce new information. The second condition prevents one from taking an isotherm that is too close to the laser spot, where the influence of departures from the Dirac delta behavior of the laser pulse is significant.

Once the isotherms are selected, their shape is approximated resorting to the already described least-squares procedure. The results of this procedure are the coordinates of the center and the length of both semi-axes of the ellipse (c.f., Eq. 12 ). Knowing the ratio of the semi-axes, the ratio of thermal conductivities $k_{y}$ is calculated using Eq. 10. The experience gathered in the course of this research has shown that random reflections of the laser beam may take place. The result is the presence of some hot spots on the measurement surface of the material. To eliminate such cases, the isotherms producing $k_{y}$ that differ by more than $\pm 20 \%$ from the expected value of the material $k_{y}$ are discarded. 
Fig. 8 Retrieved thermal conductivity based on inverse analysis

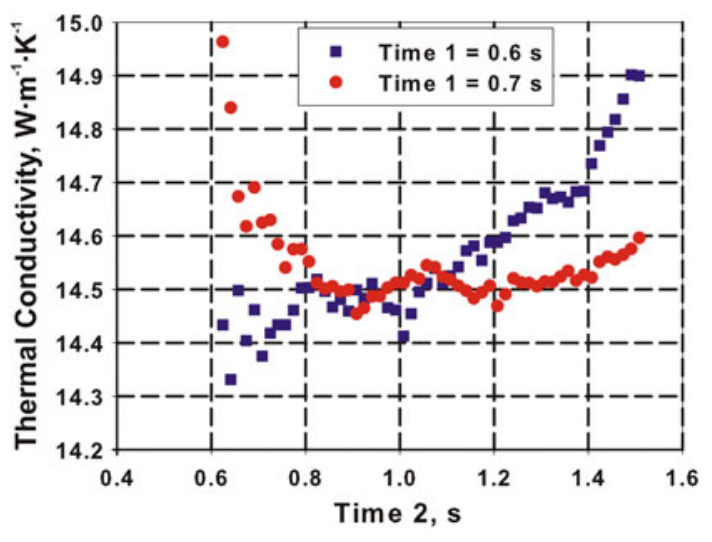

For each time $t_{1}$, the second time instant $t_{2}$ needs to be found. As shown in Fig. 3, the temporal variation of temperature at a given point assumes a maximum. Numerous tests supported by sensitivity analysis have shown that the best accuracy of the evaluated diffusivity is achieved when the times $t_{1}$ and $t_{2}$ are located on opposite sides of this maximum. Starting from that maximum, the time $t_{2}$ is sampled every $0.1 \mathrm{~s}$ until the value of the temperature is $3 \mathrm{~K}$ above the initial temperature. As already mentioned, smaller values of the temperature at $t_{2}$ introduce excessive error. The behavior of the error is shown in Fig. 8 showing a sample plot of the obtained thermal diffusivities evaluated taking two values of the first time instant $t_{1}$ and various times $t_{2}$. It can readily be seen that within a certain range of times, the value of the thermal conductivity (diffusivity) remains constant. Times smaller than that range produce bad results due to the departure of the heat source from the Dirac delta behavior, while at larger times both the deteriorating accuracy of measurements and the influence of the heat exchange with the environment can be seen.

The question of the influence of the dependence on temperature of the diffusivity requires additional comments. Although at the point where the laser beam impinges the MS the temperature may rise by $80 \mathrm{~K}$, the region of this temperature is very small and even in this domain, the temperature decays rapidly. As additionally the measurements of the temperature field are taken at a certain distance from the impingement point and at later times, the influence of the changes in material properties are marginal.

The next step of the procedure of retrieving the diffusivity is to evaluate this quantity from Eq. 13, taking each selected pairs of times $t_{1}$ and $t_{2}$. As in the case of determining the ratio of the conductivities, a Bayesian approach is applied. Values exceeding the expected values of the diffusivity by $30 \%$ are discarded. The final value of the diffusivity is then taken as the mean value of the remaining diffusivity values.

The question of calculating the values of the initial temperature requires additional comments. When the sample is for a longer time in contact with the environment of constant temperature, the initial temperature should be uniform on the entire MS. In practice, as the emissivity of the material is not completely uniform, the initial temperature recorded by the camera is not constant. This is especially true when a carbonaceous material with inclusions of graphite is investigated. As a result, although the initial temperature is homogeneous, the IR camera shows small temper- 
ature differences. It should be stressed that the temperature pairs taken in the process of determination of the diffusivity are measured at the same locations. As the algorithm uses a ratio of temperature excess at the same points, the influence of the local value of the emissivity cancels out. To account for the differences in local emissivities, the measured initial temperature is not treated as a constant, but the value of $T_{\text {init }}=T_{\text {measured }}\left(x_{i}, y_{i}, z=0, t=0\right)$ is taken when evaluating $\Theta_{\text {measured }}$ in Eq. 13.

It should be stressed that heuristic elements of the procedure are limited solely to the selection of the values of the isotherms and time instants at which the temperature field is sampled. However, the developed technique does not select these values blindly. Although the isotherms to evaluate $k_{y}$ are taken randomly, only values that satisfy some obvious, described above rules, are finally used in the calculations. The evaluation of the diffusivity is carried out for a certain number of pairs of sampling times selected also in a random way. Some of the results are discarded using common sense rules described above. The final result is obtained by taking an average of the remaining results. The randomness in the algorithm and averaging reduces the influence of a systematic error that might be introduced by flaws and cracks in the MS. As the device is designed to be used in an industrial environment, the presence of such flaws should be accounted for.

The Bayesian elements of the procedure can be summed up such that an approximate value of the determined values of $k_{y}$ and $D_{x}$ is used to discard intermediate results. This is in line with the Bayesian philosophy, where each a priori information on the phenomenon under investigation enhances the accuracy of the mathematical description. The measurement technique is developed to work in an industrial environment for the purpose of quality control. Under such conditions, the order of the magnitude of the material properties is always known. Should the technique be applied to a completely new material, a very wide interval of the predicted values of the properties can be predicted. Under such an assumption, this constraint would become inactive. In a lab environment with a smooth MS, the application of the heuristics turned out to be superfluous.

\section{Repeatability and Comparison with Other Methods}

Using one set of recorded temperatures, the technique produces several values of the diffusivities. After the results laden by significant error are discarded, the obtained results are within a $5 \%$ error margin. Table 1 shows comparisons of results obtained by other methods: the commercial Parker flash technique, the static comparison method, and the present technique. As both the Parker and the present technique yield the thermal diffusivity, for the purpose of comparison this property has been converted into the thermal conductivity using a known density and specific heat. The manufacturer declares that the error margin of both benchmark techniques is of the order of $5 \%$.

To check the stability of the proposed technique, a set of measurements has been carried out for anisotropic carbonaceous materials of various thermal conductivities. The thermal properties of these materials are shown in Table 2. The diffusivity measurements have been carried out using the standard Parker method. 
Table 1 Benchmark data

\begin{tabular}{ll}
\hline Method & $\begin{array}{l}\text { Thermal conductivity } \\
\left(\mathrm{W} \cdot \mathrm{m}^{-1} \cdot \mathrm{K}^{-1}\right)\end{array}$ \\
\hline Parker flash method & 40.6 \\
Static comparison method & 43.1 \\
In-house technique & 41.2 \\
\hline
\end{tabular}

Table 2 Benchmark data of carbonaceous material

\begin{tabular}{lll}
\hline Material & $D_{x}\left(\mathrm{~m}^{2} \cdot \mathrm{s}^{-1}\right)$ & $D_{y}\left(\mathrm{~m}^{2} \cdot \mathrm{s}^{-1}\right)$ \\
\hline Mat-1 & $5.11 \times 10^{-6}$ & $5.21 \times 10^{-6}$ \\
Mat-2 & $6.29 \times 10^{-6}$ & $6.47 \times 10^{-6}$ \\
Mat-3 & $1.01 \times 10^{-5}$ & $1.06 \times 10^{-5}$ \\
\hline
\end{tabular}

Table 3 Thermal diffusivities evaluated for Mat-1 (TC $5 \mathrm{~W} \cdot \mathrm{m}^{-1} \cdot \mathrm{K}^{-1}$ )

\begin{tabular}{lllll}
\hline Measurement point & $D_{x}\left(\mathrm{~m}^{2} \cdot \mathrm{s}^{-1}\right)$ & $D_{y}\left(\mathrm{~m}^{2} \cdot \mathrm{s}^{-1}\right)$ & Error in $D_{x}(\%)$ & Error in $D_{y}(\%)$ \\
\hline 1 & $5.04 \times 10^{-6}$ & $5.19 \times 10^{-6}$ & 1.5 & 0.4 \\
2 & $5.25 \times 10^{-6}$ & $5.23 \times 10^{-6}$ & 2.6 & 0.4 \\
3 & $5.39 \times 10^{-6}$ & $5.25 \times 10^{-6}$ & 5.2 & 0.7 \\
4 & $4.97 \times 10^{-6}$ & $5.04 \times 10^{-6}$ & 2.9 & 3.4 \\
5 & $5.11 \times 10^{-6}$ & $5.19 \times 10^{-6}$ & 0.0 & 0.4 \\
Mat-1 & $5.11 \times 10^{-6}$ & $5.21 \times 10^{-6}$ & & \\
\hline
\end{tabular}

Table 4 Thermal diffusivities evaluated for Mat-2 (TC in the range ( 8 to 12$) \mathrm{W} \cdot \mathrm{m}^{-1} \cdot \mathrm{K}^{-1}$ )

\begin{tabular}{lllll}
\hline Measurement point & $D_{x}\left(\mathrm{~m}^{2} \cdot \mathrm{s}^{-1}\right)$ & $D_{y}\left(\mathrm{~m}^{2} \cdot \mathrm{s}^{-1}\right)$ & Error in $D_{x}(\%)$ & Error in $D_{y}(\%)$ \\
\hline 1 & $6.22 \times 10^{-6}$ & $6.64 \times 10^{-6}$ & 1.1 & 2.7 \\
2 & $6.51 \times 10^{-6}$ & $6.60 \times 10^{-6}$ & 3.6 & 2.0 \\
3 & $6.27 \times 10^{-6}$ & $6.36 \times 10^{-6}$ & 0.3 & 1.7 \\
4 & $6.45 \times 10^{-6}$ & $6.57 \times 10^{-6}$ & 2.6 & 1.7 \\
5 & $.76 \times 10^{-6}$ & $6.99 \times 10^{-6}$ & 7.5 & 8.6 \\
Mat-2 & $6.29 \times 10^{-6}$ & $6.47 \times 10^{-6}$ & & \\
\hline
\end{tabular}

For all benchmark material measurements have been performed at five different locations on the external surface of the sample. The measured diffusivities are listed in Tables 3, 4, and 5. The error in the tables are with respect to the benchmark values. The differences between Parker's and the developed techniques are smaller for materials of lower conductivity, where the error was below $2 \%$. Materials of higher diffusivities produce larger errors but still they are of the order of $5 \%$. The deterioration of the accuracy of the measurements might be attributed to the rapid temperature changes in 
Table 5 Thermal diffusivities evaluated for Mat-3 (TC in the range (15 to 19) $\mathrm{W} \cdot \mathrm{m}^{-1} \cdot \mathrm{K}^{-1}$ )

\begin{tabular}{lllll}
\hline Measurement point & $D_{x}\left(\mathrm{~m}^{2} \cdot \mathrm{s}^{-1}\right)$ & $D_{y}\left(\mathrm{~m}^{2} \cdot \mathrm{s}^{-1}\right)$ & Error in $D_{x}(\%)$ & Error in $D_{y}(\%)$ \\
\hline 1 & $9.81 \times 10^{-6}$ & $1.06 \times 10^{-5}$ & 2.8 & 0.3 \\
2 & $9.48 \times 10^{-6}$ & $1.04 \times 10^{-5}$ & 6.0 & 1.5 \\
3 & $9.30 \times 10^{-6}$ & $1.03 \times 10^{-5}$ & 7.8 & 2.8 \\
4 & $9.54 \times 10^{-6}$ & $1.03 \times 10^{-5}$ & 5.4 & 2.6 \\
5 & $9.42 \times 10^{-6}$ & $1.02 \times 10^{-5}$ & 6.6 & 3.5 \\
Mat-3 & $1.02 \times 10^{-6}$ & $1.06 \times 10^{-5}$ & & \\
\hline
\end{tabular}

better conductors. To record such fields, an IR camera of higher frequency should be applied.

\section{Conclusions}

The developed non-destructive and rapid technique proved to be reliable and robust. The accuracy of the technique is comparable with the standard Parker's technique widely used in practice. The advantage od the proposed approach when compared to the standard Parker's technique is the non-destructive character of the former. Moreover, the proposed method can readily be applied to determine thermal diffusivities of orthotropic crystalline media and equivalent diffusivities of composites, if only the main axis of the thermal-conductivity tensor is co-planar with the surface of the sample. The material sample should be large enough to be treated, within time intervals of the order of a few seconds, such as a semi-infinite medium. The excitation of the temperature field is accomplished by a laser flash, treated in the model as Dirac's function in time and space. Thus, it is important that the duration of the pulse is short and the heated surface area is small. As the ellipticity of the isotherms is used to retrieve the ratio of the principal thermal conductivities, the shape of the heated area should be circular. The temperature field is recorded by an IR camera of appropriate space and time resolution. The duration of the experiment is of the order of a few seconds and the data processing of a single measurement is of the order of a minute. Thus, the technique can be used in the on-line quality control for processes where the thermal diffusivity (conductivity) is an important indicator of the quality of the product. The tests carried out so far, show that the accuracy of the method is sufficient for engineering applications.

The accuracy slightly deteriorates with the increased conductivity of the material under investigation. Work is in progress to mitigate this discrepancy. Initial results show that better temporal resolution of the camera would cure this problem. Other investigated improvements of the technique encompass more sophisticated models of the heat transfer of the sample, in order to account for heat losses due to convection and radiation. Another open question that requires in-depth examination is the optimal choice of the sampling times and values of isotherms. Appropriate research based on 
numerical simulations accounting for heat convection and radiation on the MS has already been started.

As already mentioned, good heat conductors are difficult to handle using cameras of low time resolution. The reason for this is the rapid temperature equilibration after instantaneous heating. In such media, very soon after the flash, the temperature becomes homogeneous. Two points need to be mentioned here. The first snapshot of the temperature field can be taken only after the laser head is removed from the field of view of the camera. If the process of equilibration of the temperature is very fast, the temperature differences are too small to produce reasonable accuracy. Moreover, the technique measures the excess of the temperature over the initial one. For good conductors, the available temperature range can be not high enough for exact measurement. For the installation at hand, the upper limit of thermal conductivity that can be measured is of the order of $50 \mathrm{~W} \cdot \mathrm{m}^{-1} \cdot \mathrm{K}^{-1}$.

The technique has been used at room temperatures and applied to materials of thermal conductivity in the range of $5 \mathrm{~W} \cdot \mathrm{m}^{-1} \cdot \mathrm{K}^{-1}$ to $40 \mathrm{~W} \cdot \mathrm{m}^{-1} \cdot \mathrm{K}^{-1}$. A heuristic recommendation concerning the times and locations where the temperature field should be measured is included. The idea and the measurement device is the subject of a patent application [1].

Open Access This article is distributed under the terms of the Creative Commons Attribution License which permits any use, distribution, and reproduction in any medium, provided the original author(s) and the source are credited.

\section{Appendix 1: Measurement Equipment}

Laser

The laser used in the tests was a diode laser manufactured by IPG Photonics. The energy of the impulse was in the range of $10 \mathrm{~W}$ to $200 \mathrm{~W}$; the duration of the laser impulse was kept in the range of $0.2 \mathrm{~s}$ to $0.5 \mathrm{~s}$ controlled by $5 \mathrm{VDC}$. The device is controlled using the RS232 communication protocol.

\section{IR Camera}

An off-the shelf IR camera manufactured by FLIR, with a resolution of 16-bit $320 \times$ 240 pixels was used. The recording frequency at the highest resolution is $60 \mathrm{~Hz}$. The IR camera is controlled using FLIR LabVIEW ThermoVision 3.0 library.

\section{In-House Controller}

It is used for protecting laser against unexpected laser emission and controlling the ventilation of the measuring head. The device is controlled using RS232 communication protocol. 


\section{Step Motor Controller}

This device is used for controlling the operation of the step motor system, which is responsible for moving the laser head. The device is controlled using RS232 communication protocol.

\section{Appendix 2: Uncertainty Analysis}

The temperature measurement is inherently associated with several uncertainty factors. Thus, the interpretation of the experimental data should be accompanied by an uncertainty analysis.

For the IR camera used in the experiments, the factors which influence the level of measurement uncertainty are: thermogram acquisition rate, surface emissivity, accuracy of the IR camera, and the size of the pixel.

The time, when the first temperature field is recorded after the laser shot produces a temperature uncertainty $u_{t}$ which is associated with the IR camera acquisition rate. The recorded frequency in the experiment was around 60 frames per second, so the acquisition rate $\Delta t$ is equal to $0.017 \mathrm{~s}$. The biggest recorded temperature difference was $\Delta T=3.77 \mathrm{~K}$ within one second. The temperature uncertainty due to the acquisition rate can be calculated as $u_{t}=\frac{\partial T}{\partial t} \Delta t$, where the partial derivative can be approximated by a difference quotient as $\frac{\Delta T}{\Delta t}$ and is equal to $3.77 \mathrm{~K} \cdot \mathrm{s}^{-1}$. The calculated uncertainty was thus $u_{t}=0.064 \mathrm{~K}$. The quality of the camera detector is the second source of measurement uncertainty. The minimal temperature difference distinguished by the camera is $0.1 \mathrm{~K}$ which defines the detector uncertainty $u_{c}$. The next contribution of the uncertainty comes from the emissivity of the measured surface. For the lab tests used to compare with standard techniques, the surfaces were smooth and black painted. The uncertainty in emissivity $u_{\epsilon}$ is assessed to be of the order of 0.01 . The additional contribution of uncertainty is associated with the pixel location $u_{x}$ which is of the order of $0.01 \mathrm{~mm}$, i.e., and the location uncertainty is equal to the dimension of one pixel. The total uncertainty $u_{T}$, of the temperature field measurement results from the law of error propagation [20] can be calculated as

$$
\begin{aligned}
u_{T}^{2}= & \left(\frac{\partial T}{\partial T_{\mathrm{c}}}\right)^{2} u_{\mathrm{c}}^{2}+\left(\frac{\partial T}{\partial \varepsilon}\right)^{2} u_{\varepsilon}^{2}+\left(\frac{\partial T}{\partial x}\right)^{2} u_{x}^{2}+\left(\frac{\partial T}{\partial t}\right)^{2} u_{t}^{2} \\
& +2 \frac{\partial T}{\partial T_{\mathrm{c}}} \frac{\partial T}{\partial \varepsilon} \operatorname{cov}\left(T_{\mathrm{c}}, \varepsilon\right)+2 \frac{\partial T}{\partial T_{\mathrm{c}}} \frac{\partial T}{\partial x} \operatorname{cov}\left(T_{\mathrm{c}}, x\right)+2 \frac{\partial T}{\partial T_{\mathrm{c}}} \frac{\partial T}{\partial t} \operatorname{cov}\left(T_{\mathrm{c}}, t\right) \\
& +2 \frac{\partial T}{\partial \varepsilon} \frac{\partial T}{\partial x} \operatorname{cov}(\epsilon, x)+2 \frac{\partial T}{\partial \varepsilon} \frac{\partial T}{\partial t} \operatorname{cov}(\varepsilon, t)+2 \frac{\partial T}{\partial x} \frac{\partial T}{\partial t} \operatorname{cov}(x, \varepsilon)
\end{aligned}
$$

where subscript $\mathrm{c}$ refers to the infrared camera uncertainty, $x$ and $t$ to a pixel location and time uncertainty, while $\epsilon$ is associated with the surface emissivity factor. The covariances denoted as "cov" account for the correlation between two parameters. In the considered case, these quantities are not correlated, so the covariances vanish and the equation simplifies to 


$$
u_{T}=\left[\left(\frac{\partial T}{\partial T_{\mathrm{c}}}\right)^{2} u_{\mathrm{c}}^{2}+\left(\frac{\partial T}{\partial \varepsilon}\right)^{2} u_{\epsilon}^{2}+\left(\frac{\partial T}{\partial x}\right)^{2} u_{x}^{2}+\left(\frac{\partial T}{\partial t}\right)^{2} u_{t}^{2}\right]^{0.5}
$$

The partial derivatives in the above are determined from the recorded temperature fields and approximated by difference quotients. The evaluated derivatives $\frac{\partial T}{\partial T_{\mathrm{c}}}, \frac{\partial T}{\partial x}, \frac{\partial T}{\partial \epsilon}, \frac{\partial T}{\partial t}$ were equal to $1,8,0.5$, and 3.77 , respectively. The total uncertainty of the measured temperature $u_{T}$, is $0.57 \mathrm{~K}$.

It should be stressed that the value is a pessimistic approximation of the uncertainty. The influence of the error in the emissivities is in fact negligible. The reason being that the experimental values used in the evaluation of the properties are ratios of temperature differences, rather than temperatures alone.

\section{References}

1. W. Adamczyk, R. Bialecki, T. Kruczek, Patent Application, EP 11195498.8, 23 Dec 2011

2. R.C. Aster, B. Borchers, C.H. Thurber, Parameter Estimation and Inverse Problems (Elsevier, Amsterdam, 2005)

3. T. Baba, A. Ono, Meas. Sci. Technol. 12, 2046 (2001)

4. R.A. Biaecki, Z. Buliski, in Encyclopedia of Thermal Stress, ed. by R.H. Hetnarski (Springer, Berlin, 2013) (in press)

5. P.G. Bison, S. Marinetti, E. Grinzato, in 5th International Conference on Quantitative Infrared Thermography, Champagne-Ardenne, 2000

6. F. Cernusci, P.G. Bison, A. Figari, S. MArinetti, E. Grinzato, Int. J. Thermophys. 25, 439 (2004)

7. W. Buck, S. Rudtsch, in Springer Handbook of Material Measurements, ed. by H. Czichos, T. Saito, L. Smit (Springer, Heidelberg, 2006)

8. K.D. Cole, Sheikh A. Haji, Heat Conduction Using Green's Function (CRC Press, Taylor and Francis Group, Boca Raton, 2011)

9. D. Demange, P. Beauchene, M. Bejet, Revue Gen. Thermique 36, 755 (1997)

10. E1461-01, ASTM Test Method. Standard Test Method for Thermal Diffusivity of Solids by the Flash Method. Annual Book of ASTM Standards (American Society for Testing and Materials, West Conshohocken, PA, 2001)

11. B. Friedman, Principles and Techniques of Applied Mathematics (Dover Publications, New York, 1990)

12. A. Mandelis, M. Munidasa, Non-contact photothermal method for diffusivity and electronic defect properties of solids, US Patent 5667300, 1997

13. F. Lakestani, A. Salerno, A. Volcan, US Patent 20060153269, 2006

14. C. Meola, G.M. Carlomagno, A. Squillace, A. Vitiello, Eng. Fail. Anal. 13, 380 (2006)

15. H.R.B. Orlande, M.N. Ozisik, Inverse Heat Transfer, Fundamentals and Applications (Taylor and Francis, New York, 2000)

16. J.W. Parker, R.J. Jenkins, C.P. Butler, G.L. Abbott, J. Appl. Phys. 32, 1679 (1961)

17. W.H. Press, S.A. Teukolsky, W.T. Vetterling, B.P. Flannery, Numerical Recipes (Cambridge University Press, Cambridge, 2007)

18. J.G. Sun, Method for Thermal Tomography of Thermal Effusivity from Pulsed Thermal Imaging, US Patent 7,365,330, 2008

19. O. Yu. Troitsky, H. Reiss, Int. J. Thermophys. 30, 1283 (2012)

20. K. Woodbury (ed.), Inverse Engineering Handbook (CRC Press, Boca Raton, 2003) 